\title{
MOCK THETA FUNCTIONS AND QUANTUM MODULAR FORMS
}

\author{
AMANDA FOLSOM ${ }^{1}, \mathrm{KEN} \mathrm{ONO}^{2}$ and ROBERT C. RHOADES ${ }^{3,4}$ \\ ${ }^{1}$ Department of Mathematics, Yale University, New Haven, CT 06520, USA; \\ email: amanda.folsom@yale.edu \\ ${ }^{2}$ Department of Mathematics, Emory University, Atlanta, GA 30322, USA; \\ email: ono@mathcs.emory.edu \\ ${ }^{3}$ Department of Mathematics, Stanford University, Stanford, CA 94305, USA; \\ email: rhoades@math.stanford.edu,rob.rhoades@gmail.com \\ ${ }^{4}$ Current address: Center for Communications Research, Princeton, NJ 08540, USA
}

Received 1 November 2012; accepted 23 August 2013

\begin{abstract}
Ramanujan's last letter to Hardy concerns the asymptotic properties of modular forms and his 'mock theta functions'. For the mock theta function $f(q)$, Ramanujan claims that as $q$ approaches an even-order $2 k$ root of unity, we have

$$
f(q)-(-1)^{k}(1-q)\left(1-q^{3}\right)\left(1-q^{5}\right) \cdots\left(1-2 q+2 q^{4}-\cdots\right)=O(1) .
$$

We prove Ramanujan's claim as a special case of a more general result. The implied constants in Ramanujan's claim are not mysterious. They arise in Zagier's theory of 'quantum modular forms'. We provide explicit closed expressions for these 'radial limits' as values of a 'quantum' $q$-hypergeometric function which underlies a new relationship between Dyson's rank mock theta function and the Andrews-Garvan crank modular form. Along these lines, we show that the Rogers-Fine false $\vartheta$-functions, functions which have not been well understood within the theory of modular forms, specialize to quantum modular forms.
\end{abstract}

2010 Mathematics Subject Classification: 11F99 (primary); 11F37, 33D15 (secondary)

\section{Overview}

In his 1920 deathbed letter to Hardy, Ramanujan gave examples of 17 curious $q$-series he referred to as 'mock theta functions' [11]. In the decades following Ramanujan's death, mathematicians were unable to determine how these functions fit into the theory of modular forms, despite

(C) The Author(s) 2013. The online version of this article is published within an Open Access environment subject to the conditions of the Creative Commons Attribution licence <http://creativecommons.org/licenses/by/3.0/>. 
their rather ubiquitous nature. Finally, the 2002 Ph.D. thesis of Zwegers [45] showed that while the mock theta functions were not modular, they could be 'completed' to produce real analytic vector-valued modular forms. Zwegers's breakthrough catalyzed the development of the overarching theory of 'weak Maass forms' by Bringmann, Ono, and collaborators [15, 16, 35, 42]. Ramanujan's mock theta functions, it turns out, are examples of 'holomorphic parts' of weak Maass forms, originally defined by Bruinier and Funke [18].

While the theory of weak Maass forms has led to a flood of applications in many disparate areas of mathematics (see [35, 42] and references therein), it is still not the case that we fully understand the deeper framework surrounding the contents of Ramanujan's last letter to Hardy. Here, we revisit Ramanujan's original claims and motivations. His last letter summarizes asymptotic properties near roots of unity of modular 'Eulerian' series. Ramanujan asks whether other Eulerian series with similar asymptotics are necessarily the sum of a modular theta function and a function which is $O(1)$ at all roots of unity. He writes: 'The answer is it is not necessarily so ... I have not proved rigorously that it is not necessarily so ... But I have constructed a number of examples ... '. In fact, Ramanujan's sole example and claim pertain to his third-order mock theta function $f(q)$.

CLAIM (Ramanujan [11]). As q approaches an even-order $2 k$ root of unity, we have

$$
f(q)-(-1)^{k}(1-q)\left(1-q^{3}\right)\left(1-q^{5}\right) \cdots\left(1-2 q+2 q^{4}-\cdots\right)=O(1) .
$$

Here, we prove (in Theorem 1.1) Ramanujan's claim as a special case of a more general result (Theorem 1.2). We provide an explicit closed formula for the implied constants in Ramanujan's claim, and show that they are values of a 'quantum' $q$-hypergeometric function which underlies a new relationship between Dyson's rank mock theta function and the Andrews-Garvan crank modular form, two of the most studied $q$-series in the theory of partitions (see, for example, [5, 8, $16,23,33])$.

In this paper, it is the new theory of quantum modular forms that serves as a foundation for our main theorems. As defined by Zagier [43], a quantum modular form is (loosely) a $\mathbb{C}$-valued function defined on $\mathbb{Q}$ that exhibits usual modular transformation properties, up to the addition of a 'suitably' continuous or analytic function. A crucial property we use is that quantum modular forms make it possible to pass between the upper and lower halves of the complex plane. In doing so, we are led to the Rogers-Fine 'false' $\vartheta$-functions, functions which Ramanujan claimed 'do not enter into mathematics as beautifully as the ordinary theta functions.' On the contrary, we prove (in Theorem 1.3) that the Rogers-Fine false $\vartheta$-functions specialize to quantum modular forms, and that Ramanujan's own mock functions play key roles.

\section{Introduction and statement of results}

Ramanujan's enigmatic last letter to Hardy [11] gave tantalizing hints of his theory of mock theta functions. Thanks to Zwegers [44, 45], it is now known that these functions are specializations of nonholomorphic Jacobi forms. They are holomorphic parts of certain weight-1/2 harmonic weak Maass forms of Bruinier and Funke [18]. This realization has many applications in combinatorics, number theory, physics, and representation theory (for example, see [35, 42]).

Here, we revisit Ramanujan's original claims and motivation from his deathbed letter [11]: 
'... I discovered very interesting functions recently which I call "Mock" $\vartheta$-functions. Unlike the "False" $\vartheta$-functions (studied partially by Prof. Rogers in his interesting paper) they enter into mathematics as beautifully as the ordinary theta functions....'

The next page of the letter summarizes the asymptotic properties, near roots of unity, of Eulerian series (also known as $q$-hypergeometric series) which are modular theta functions. He then asks whether other Eulerian series with similar asymptotics are necessarily the sum of a modular theta function and a function which is $O(1)$ at all roots of unity. He writes:

'The answer is it is not necessarily so. When it is not so I call the function Mock $\vartheta$-function. I have not proved rigorously that it is not necessarily so. But I have constructed a number of examples in which it is inconceivable to construct a $\vartheta$-function to cut out the singularities of the original function.'

REMARK. Griffin, the second author, and Rolen [28] have confirmed Ramanujan's speculation. There are no weakly holomorphic modular forms which exactly cut out the singularities of Ramanujan's mock theta functions.

The only specific example Ramanujan offers pertains to the $q$-hypergeometric function

$$
f(q):=1+\frac{q}{(1+q)^{2}}+\frac{q^{4}}{(1+q)^{2}\left(1+q^{2}\right)^{2}}+\cdots .
$$

This function is convergent for $|q|<1$ and those roots of unity $q$ with odd order. For even-order roots of unity, $f(q)$ has exponential singularities. For example, as $q \rightarrow-1$, we have

$$
\begin{gathered}
f(-0.994) \sim-1.08 \cdot 10^{31}, \quad f(-0.996) \sim-1.02 \cdot 10^{46}, \\
f(-0.998) \sim-6.41 \cdot 10^{90} .
\end{gathered}
$$

To cancel the exponential singularity at $q=-1$, Ramanujan found the function $b(q)$, which is modular (Here, $q^{-\frac{1}{24}} b(q)$ is modular with respect to $z$, where $q:=e^{2 \pi i z}$.) up to multiplication by $q^{-\frac{1}{24}}$, defined in his notation by

$$
b(q):=(1-q)\left(1-q^{3}\right)\left(1-q^{5}\right) \cdots\left(1-2 q+2 q^{4}-\cdots\right) .
$$

The exponential behavior illustrated above is canceled in the numerics below.

\begin{tabular}{|c||l|l|l|l|l|}
\hline$q$ & -0.990 & -0.992 & -0.994 & -0.996 & -0.998 \\
\hline$f(q)+b(q)$ & $3.961 \ldots$ & $3.969 \ldots$ & $3.976 \ldots$ & $3.984 \ldots$ & $3.992 \ldots$ \\
\hline
\end{tabular}


It appears that $\lim _{q \rightarrow-1}(f(q)+b(q))=4$. More generally, as $q$ approaches an even-order $2 k$ root of unity, Ramanujan claimed that

$$
f(q)-(-1)^{k} b(q)=O(1) .
$$

REMARK. In his survey of Ramanujan's 'lost notebook' [10], Berndt writes eloquently about this claim and Ramanujan's imprecise definition of a mock theta function.

REMARK. Ramanujan's last letter also inspired the problem of determining the asymptotics of the coefficients of mock theta functions such as $f(q)$. Andrews [1] and Dragonette [22] obtained asymptotics for the coefficients of $f(q)$, and Bringmann and the second author [15] later obtained an exact formula for these coefficients.

Watson [40] was the first to prove Ramanujan's claim about $f(q)$. We provide a new proof of Ramanujan's claim. Moreover, we obtain a simple closed formula for the suggested $O(1)$ constants as values of a 'quantum' $q$-hypergeometric series.

THEOREM 1.1. If $\zeta$ is a primitive even-order $2 k$ root of unity, then, as $q$ approaches $\zeta$ radially within the unit disk, we have that

$$
\lim _{q \rightarrow \zeta}\left(f(q)-(-1)^{k} b(q)\right)=-4 \cdot \sum_{n=0}^{k-1}(1+\zeta)^{2}\left(1+\zeta^{2}\right)^{2} \cdots\left(1+\zeta^{n}\right)^{2} \zeta^{n+1} .
$$

EXAMPLE. Since empty products equal 1, Theorem 1.1 confirms that $\lim _{q \rightarrow-1}(f(q)+b(q))=4$.

EXAMPLE. For $k=2$, Theorem 1.1 gives $\lim _{q \rightarrow i}(f(q)-b(q))=4 i$. The table below nicely illustrates this fact:

\begin{tabular}{|c||l|l|l|}
\hline$q$ & $0.992 i$ & $0.994 i$ & $0.996 i$ \\
\hline$f(q)$ & $\sim 1.9 \cdot 10^{6}-4.6 \cdot 10^{6} i$ & $\sim 1.6 \cdot 10^{8}-3.9 \cdot 10^{8} i$ & $\sim 1.0 \cdot 10^{12}-2.5 \cdot 10^{12} i$ \\
\hline$f(q)-b(q)$ & $\sim 0.0577+3.855 i$ & $\sim 0.0443+3.889 i$ & $\sim 0.0303+3.924 i$ \\
\hline
\end{tabular}

REMARK. The values of $f(q)$ at odd-order roots of unity are well defined, and can be easily computed directly from (1.1).

It turns out that Theorem 1.1 is a special case of a much more general theorem, one which surprisingly relates two of the most famous $q$-series in the theory of partitions. To make it precise, we require Dyson's rank function $R(w ; q)$, the Andrews-Garvan crank function $C(w ; q)$, and the recently studied 
$q$-hypergeometric series $U(w ; q)$. The $q$-series $R(w ; q)$ and $C(w ; q)$ are among the most important generating functions in the theory of partitions. These famous series play a prominent role in the study of integer partition congruences (for example, see $[5,8,16,23,33])$.

To define these series, throughout we let $(a ; q)_{0}:=1$ and

$$
(a ; q)_{n}:= \begin{cases}(1-a)(1-a q)\left(1-a q^{2}\right) \cdots\left(1-a q^{n-1}\right) & \text { if } n \in \mathbb{Z}^{+}, \\ (1-a)(1-a q)\left(1-a q^{2}\right) \cdots & \text { if } n=\infty .\end{cases}
$$

Dyson's rank function is given by

$$
R(w ; q)=\sum_{n=0}^{\infty} \sum_{m \in \mathbb{Z}} N(m, n) w^{m} q^{n}:=1+\sum_{n=1}^{\infty} \frac{q^{n^{2}}}{(w q ; q)_{n} \cdot\left(w^{-1} q ; q\right)_{n}} .
$$

Here, $N(m, n)$ is the number of partitions of $n$ with rank $m$, where the rank of a partition is defined to be its largest part minus the number of its parts. If $w \neq 1$ is a root of unity, then it is known that $R(w ; q)$ is (up to a power of $q$ ) a mock theta function (i.e., the holomorphic part of a weight-1/2 harmonic Maass form) (for example, see [16]). The Andrews-Garvan crank function is defined by

$$
C(w ; q)=\sum_{n=0}^{\infty} \sum_{m \in \mathbb{Z}} M(m, n) w^{m} q^{n}:=\frac{(q ; q)_{\infty}}{(w q ; q)_{\infty} \cdot\left(w^{-1} q ; q\right)_{\infty}} .
$$

Here, $M(m, n)$ is the number of partitions of $n$ with crank $m$ [5]. For roots of unity $w, C(w ; q)$ is (up to a power of $q$ ) a modular form.

The series $U(w ; q)$ has recently been studied by several authors $[2,7,19,37]$ in work related to unimodal sequences. This $q$-hypergeometric series is defined by

$$
U(w ; q)=\sum_{n=0}^{\infty} \sum_{m \in \mathbb{Z}} u(m, n)(-w)^{m} q^{n}:=\sum_{n=0}^{\infty}(w q ; q)_{n} \cdot\left(w^{-1} q ; q\right)_{n} q^{n+1} .
$$

Here, $u(m, n)$ is the number of strongly unimodal sequences of size $n$ with rank $m$ [19].

REMARK. In terms of the standard notation for $q$-hypergeometric series (for example, see [26, p. 4]), it turns out that $U(w ; q)$ is a ${ }_{3} \phi_{2} q$-hypergeometric series. Namely, we have that

$$
q^{-1} U(w ; q)={ }_{3} \phi_{2}\left(w q, w^{-1} q, q ; 0,0 ; q, q\right) .
$$

Theorem 1.1 is a special case of the following general theorem which relates the asymptotic behavior of these three $q$-series. Throughout, we let $\zeta_{n}:=e^{2 \pi i / n}$. 
THEOREM 1.2. Let $1 \leq a<b$ and $1 \leq h<k$ be integers with $\operatorname{gcd}(a, b)=$ $\operatorname{gcd}(h, k)=1$ and $b \mid k$. If $h^{\prime}$ is an integer satisfying $h h^{\prime} \equiv-1(\bmod k)$, then, as $q$ approaches $\zeta_{k}^{h}$ radially within the unit disk, we have that

$$
\lim _{q \rightarrow \zeta_{k}^{h}}\left(R\left(\zeta_{b}^{a} ; q\right)-\zeta_{b^{2}}^{-a^{2} h^{\prime} k} C\left(\zeta_{b}^{a} ; q\right)\right)=-\left(1-\zeta_{b}^{a}\right)\left(1-\zeta_{b}^{-a}\right) \cdot U\left(\zeta_{b}^{a} ; \zeta_{k}^{h}\right) .
$$

Five remarks.

(1) There is an integer $c(a, b, h, k)$ such that the limit in Theorem 1.2 reduces to the finite sum

$$
-\left(1-\zeta_{b}^{a}\right)\left(1-\zeta_{b}^{-a}\right) \sum_{n=0}^{c(a, b, h, k)}\left(\zeta_{b}^{a} \zeta_{k}^{h} ; \zeta_{k}^{h}\right)_{n} \cdot\left(\zeta_{b}^{-a} \zeta_{k}^{h} ; \zeta_{k}^{h}\right)_{n} \cdot \zeta_{k}^{h(n+1)}
$$

(2) Theorem 1.1 is the $a=1$ and $b=2$ case of Theorem 1.2 because $R(-1 ; q)=$ $f(q)$, combined with the well-known fact that $C(-1 ; q)=b(q)$.

(3) A variant of Theorem 1.2 holds when $b \nmid k$. This is obtained by modifying the proof to guarantee that the two resulting asymptotic expressions match.

(4) At roots of unity where $R\left(\zeta_{b}^{a} ; q\right)$ does not have a singularity, the value can be computed directly. The remark after Theorem 1.1 is a special case of this fact. Moreover, this value is related to the value of a partial theta function. See the paper by Bringmann and the first and last authors [13] for more about the relationship between partial theta functions and mock theta functions at roots of unity where there are not singularities. Theorem 1.3 contains a result dealing with the relation between mock theta functions and partial theta functions at roots of unity where the mock theta function does not have singularities.

(5) It is natural to ask how Theorems 1.1 and 1.2 may generalize to Ramanujan's other mock theta functions. In this regard, we first note that Theorem 1.2 applies to many of Ramanujan's mock theta functions using the relationship $R(w ; q)=(1-w)+w(1-w) g_{3}(w ; q)$. Here, $g_{3}(w ; q)$ is the 'universal' mock theta function, aptly named, as it can be related to Ramanujan's original mock theta functions upon suitable specialization of its parameters (see [27]). See also recent related works by the first author [25], as well as [9], for more along these lines.

Recently, the second two authors, together with Bryson and Pitman [19], investigated the $q$-series $U(w ; q)$ in connection with the theory of quantum modular forms. Following Zagier (Zagier's definition is intentionally vague, with the idea that flexibility is required to allow for interesting examples. Here, we modify his definition to include half-integral weights $k$ and multiplier systems $\epsilon(\gamma)$.)[43], a weight $k$ quantum modular form is a complex-valued function $f$ on $\mathbb{Q}$, or possibly $\mathbb{P}^{1}(\mathbb{Q}) \backslash S$ for some set $S$, such that, for all $\gamma=\left(\begin{array}{ll}a & b \\ c & d\end{array}\right) \in \mathrm{SL}_{2}(\mathbb{Z})$, 
the function

$$
h_{\gamma}(x):=f(x)-\epsilon(\gamma)(c x+d)^{-k} f\left(\frac{a x+b}{c x+d}\right)
$$

satisfies a 'suitable' property of continuity or analyticity. The $\epsilon(\gamma)$ are suitable complex numbers, such as those in the theory of half-integral-weight modular forms when $k \in \frac{1}{2} \mathbb{Z} \backslash \mathbb{Z}$. In particular, Zagier offered a number of examples of such forms by making use of Dedekind sums, period polynomials, and a few curious $q$-series identities. Particularly interesting examples of such forms relate functions which are simultaneously defined on both $\mathbb{H}=\mathbb{H}^{+}$and $\mathbb{H}^{-}$, the upper half and lower half of the complex plane, respectively. The quantum form is the device which makes it possible to pass between the two half-planes.

Theorem 1.3 of [19] proves that $\phi(x):=e^{-\pi i x / 12} \cdot U\left(1 ; e^{2 \pi i x}\right)$ is a weight-3/2 quantum modular form on $\mathbb{H}^{+} \cup \mathbb{Q} \backslash\{0\}$ (meaning that $\phi(x)$ is quantum on $\mathbb{Q} \backslash\{0\}$, and this domain may be extended to include $\left.\mathbb{H}^{+}\right)$. Therefore, in view of the roles that $R(w ; q)$ (which is essentially a mock modular form for roots of unity $w \neq 1$ ) and $U(w ; q)$ play in Theorem 1.2, it is natural to ask about the more general relationship between mock theta functions and quantum modular forms. To this end, we seek $q$-hypergeometric series related to mock theta functions which are defined on both $\mathbb{H}^{+}$and $\mathbb{H}^{-}$. In doing so, we are led to the 'False' $\vartheta$-functions of Rogers and Fine, which Ramanujan claimed do not 'enter into mathematics as beautifully as the ordinary theta functions'.

We recall these functions. In 1917, Rogers [39] defined the important $q$-hypergeometric series

$$
F(\alpha, \beta, t ; q):=\sum_{n=0}^{\infty} \frac{(\alpha q ; q)_{n} t^{n}}{(\beta q ; q)_{n}} .
$$

This series does not typically specialize to modular forms, but instead often gives 'halves' of modular theta functions. These include many of the primary examples of 'false' and 'partial' $\vartheta$-functions. For example, we have the following special case of the work of Rogers and Fine [24]:

$$
\frac{1}{1+w} \cdot F\left(w q^{-1},-w, w ; q\right):=\frac{1}{1+w} \cdot \sum_{n=0}^{\infty} \frac{(w ; q)_{n} w^{n}}{(-w q ; q)_{n}}=\sum_{n=0}^{\infty}(-1)^{n} w^{2 n} q^{n^{2}} .
$$

Here, we consider the following specializations, where $q:=e^{2 \pi i z}$ :

$$
\begin{aligned}
& G(a, b ; z):=\frac{q^{a^{2} / b^{2}}}{1-q^{a / b}} \cdot F\left(-q^{a / b-1}, q^{a / b},-q^{a / b} ; q\right), \\
& H(a, b ; z):=q^{1 / 8} \cdot F\left(\zeta_{b}^{-a} q^{-1}, \zeta_{b}^{-a}, \zeta_{b}^{-a} q ; q^{2}\right) .
\end{aligned}
$$


We have the following false theta function identities, the second of which follows from [39, equation (1)], and the first of which is in [6].

$$
\begin{aligned}
& G(a, b ; z)=q^{a^{2} / b^{2}} \sum_{n=0}^{\infty} \frac{\left(-q^{a / b} ; q\right)_{n}}{\left(q^{a / b} ; q\right)_{n+1}} \cdot(-1)^{n} q^{n a / b}=\sum_{n=0}^{\infty}(-1)^{n} q^{(n+a / b)^{2}}, \\
& H(a, b ; z)=q^{1 / 8} \sum_{n=0}^{\infty} \frac{\left(\zeta_{b}^{-a} q ; q^{2}\right)_{n}}{\left(\zeta_{b}^{-a} q^{2} ; q^{2}\right)_{n}} \cdot\left(\zeta_{b}^{-a} q\right)^{n}=\sum_{n=0}^{\infty} \zeta_{b}^{-a n} q^{(1 / 2)(n+1 / 2)^{2}}
\end{aligned}
$$

REMARK. The second equalities in (1.10) and (1.11) are only valid for $|q|<1$.

These specializations satisfy the following nice properties often associated to quantum modular forms: convergence in $\mathbb{H}^{ \pm}$, a modular transformation law, and asymptotic expansions which are generating functions for values of $L$-functions. More precisely, we prove the following theorem.

THEOREM 1.3. Let $0<a<b$ be coprime integers, with $b$ even, and let

$$
Q_{a, b}:=\left\{\frac{h}{k} \in \mathbb{Q}: \operatorname{gcd}(h, k)=1, h>0, b \mid 2 h, b \nmid h, k \equiv a(\bmod b), k \geq a\right\} .
$$

The following are true.

(1) The functions $G(a, b ; z)$ and $H(a, b ; z)$ converge for $z \in \mathbb{H}^{+} \cup \mathbb{H}^{-}$.

(2) For $x \in Q_{a, b} \cup \mathbb{H}^{+}$, we have that

$$
G(a, b ;-x)+\frac{e^{-\pi i a / b}}{\sqrt{2 i x}} \cdot H\left(a, b ; \frac{1}{2 x}\right)=-\int_{0}^{i \infty} \frac{(-i u)^{-3 / 2} T\left(a, b ;-\frac{1}{u}\right) d u}{\sqrt{-i(u+x)}},
$$

where $T(a, b ; z)$ is a weight- 3/2 modular form defined in (2.2). That is, $G(a, b ; x)$ and $H(a, b ; x)$ are weight- $1 / 2$ quantum modular forms on $Q_{a, b} \cup \mathbb{H}^{+}$.

(3) Let $B_{r}(n)$ be the $r$ th Bernoulli polynomial. For $h / k \in Q_{a, b}$, as $t \rightarrow 0^{+}$, we have

$$
\begin{aligned}
& G\left(a, b ; \frac{-h}{k}+\frac{i t}{2 \pi}\right) \sim \sum_{r=0}^{\infty} L\left(-2 r, c_{G}\right) \cdot \frac{(-t)^{r}}{r ! \cdot b^{2 r}} \\
& H\left(a, b ; \frac{k}{2 h}+\frac{i t}{2 \pi}\right) \sim \sum_{r=0}^{\infty} L\left(-2 r, c_{H}\right) \cdot \frac{(-t)^{r}}{r ! \cdot 8^{r}}
\end{aligned}
$$

where

$$
L\left(-r, c_{G}\right)=-\frac{\left(2 k b^{2}\right)^{r}}{r+1} \sum_{n=1}^{2 k b^{2}} c_{G}(n) B_{r+1}\left(\frac{n}{2 k b^{2}}\right)
$$




$$
\begin{aligned}
& L\left(-r, c_{H}\right)=-\frac{(16 h)^{r}}{r+1} \sum_{n=1}^{16 h} c_{H}(n) B_{r+1}\left(\frac{n}{16 h}\right), \\
& c_{G}(n):= \begin{cases}\zeta_{k b^{2}}^{-h n^{2}} & \text { if } n \equiv a(\bmod 2 b), \\
-\zeta_{k b^{2}}^{-h n^{2}} & \text { if } n \equiv a+b(\bmod 2 b), \\
0 & \text { otherwise, }\end{cases} \\
& c_{H}(n):= \begin{cases}\zeta_{b}^{-a((n-1) / 2)} \zeta_{16 h}^{k n^{2}} & \text { if } n \equiv 1(\bmod 2), \\
0 & \text { otherwise. }\end{cases}
\end{aligned}
$$

Four remarks.

(1) In Section 2.1, we will prove a lemma (see Lemma 2.1) which implies, for $x=h / k \in Q_{a, b}$, that $G(a, b ;-x)$ and $H(a, b ; 1 / 2 x)$ converge. Moreover, they are explicitly given by the finite sums

$$
\begin{aligned}
G\left(a, b ;-\frac{h}{k}\right) & =\zeta_{b^{2} k}^{-2^{2} h} \sum_{n=0}^{m} \frac{\left(-\zeta_{b k}^{-a h} ; \zeta_{k}^{-h}\right)_{n}\left(-\zeta_{b k}^{-a h}\right)^{n}}{\left(\zeta_{b k}^{-a h} ; \zeta_{k}^{-h}\right)_{n+1}}, \\
H\left(a, b ; \frac{k}{2 h}\right) & =\zeta_{16 h}^{k} \sum_{n=0}^{\ell} \frac{\left(\zeta_{b}^{-a} \zeta_{2 h}^{k} ; \zeta_{h}^{k}\right)_{n}\left(\zeta_{b}^{-a} \zeta_{2 h}^{k}\right)^{n}}{\left(\zeta_{b}^{-a} \zeta_{h}^{k} ; \zeta_{h}^{k}\right)_{n}}
\end{aligned}
$$

where the nonnegative integers $\ell$ and $m$ satisfy $b(2 \ell+1)=2 h$ and $a+b m=k$, respectively.

(2) In Theorem 1.3(2), we are using the vector-valued notion of a quantum modular form.

(3) Theorem 1.2(3) gives generating functions for values of $L$-functions. Similar theorems have been previously discovered by Zagier and others (for example, see $[6,21,29,31,32,41])$.

(4) The series $G(a, b ; z)$ and $H(a, b ; z)$ are related to mock modular forms when $z \in \mathbb{H}^{-}$(see Section 2). The idea to pass between half-planes to relate mock theta functions and partial theta functions has been observed previously. For example, Zwegers observed and Lawrence and Zagier reported on such a relationship in [31]. The relationship is also discussed at length in work of the third author [38] and Mortenson [34].

EXAMPLE. Here, we will illustrate how the different parts of Theorem 1.3 may be used to understand the Rogers-Fine functions (1.10) and (1.11), and relations 
between them, at rational numbers $z$ by way of an example. If $a=1$ and $b=2$, then (1.10) and (1.11) give the identities

$$
\begin{aligned}
& G(1,2 ; z):=q^{1 / 4} \sum_{n=0}^{\infty} \frac{\left(-q^{1 / 2} ; q\right)_{n}(-1)^{n} q^{n / 2}}{\left(q^{1 / 2} ; q\right)_{n+1}}=\sum_{n=0}^{\infty}(-1)^{n} q^{(2 n+1)^{2} / 4}, \\
& H(1,2 ; z):=q^{1 / 8} \sum_{n=0}^{\infty} \frac{\left(-q ; q^{2}\right)_{n}(-q)^{n}}{\left(-q^{2} ; q^{2}\right)_{n}}=\sum_{n=0}^{\infty}(-1)^{n} q^{(2 n+1)^{2} / 8},
\end{aligned}
$$

so $G(1,2 ; z)=H(1,2 ; 2 z)$. For simplicity, we consider the rational number $h / k=1 / 1 \in Q_{1,2}$.

On one hand, from Theorem 1.3(3), we find that, asymptotically, as $t \rightarrow 0^{+}$, we have

$$
G\left(1,2 ;-1+\frac{i t}{2 \pi}\right) \sim \sum_{r=0}^{\infty} L\left(-2 r, c_{G}\right) \cdot \frac{(-t)^{r}}{r ! \cdot b^{2 r}} .
$$

We compute (using that $B_{1}(x)=x-1 / 2$ ) that $L\left(0, c_{G}\right)=-i / 2$, and so $G(1,2 ;-1) \sim-i / 2$. On the other hand, Theorem 1.3 gives $G(1,2 ;-1)$ exactly, as a finite sum, using (1.14) (see also Lemma 2.1). In particular, we have $m=0$ for $(a, b, h, k)=(1,2,1,1)$, and hence we compute that, at the root of unity -1 , the function $G(1,2 ;-1)$ is exactly equal to $G(1,2 ;-1)=\zeta_{4}^{-1} /\left(1-\zeta_{2}^{-1}\right)=$ $-i / 2$.

Similarly, using Theorem 1.3(3), we find that, asymptotically, $H\left(1,2 ; \frac{1}{2}\right) \sim$ $\zeta_{16}$. On the other hand, we may evaluate $H\left(1,2 ; \frac{1}{2}\right)$ exactly as a finite sum using (1.15) (see also Lemma 2.1). Indeed, we find that $H\left(1,2 ; \frac{1}{2}\right)=\zeta_{16}$.

We may combine these calculations with Theorem 1.3(2), to find an exact value for the integral expression appearing in Theorem 1.3(2). Namely, we have that

$$
\frac{i}{4} \int_{0}^{i \infty} \frac{(-i u)^{-\frac{3}{2}} \Theta_{1,4}\left(-\frac{1}{u}\right) d u}{\sqrt{-i(u+1)}}=\frac{i}{2}-\sqrt{\frac{i}{2}} \cdot \zeta_{16},
$$

where $\Theta_{1,4}(z):=\sum_{n \equiv 1(\bmod 4)} n q^{n^{2} / 16}$.

AMUSING REMARK. The theorems in this paper bring together some of most interesting objects which appear in Ramanujan's legacy to mathematics. Indeed, Dyson's rank, the Andrews-Garvan crank, the mock theta functions, and early examples of quantum modular forms appear as four different items in the top (These objects appear in the formulas ranked \#1, \#2,\#3, and \#5.) five of the 'ten most fascinating formulas' from Ramanujan's 'lost notebook' as tabulated [3] by Andrews and Berndt in 2008. Surprisingly, the theorems here now reveal that these objects are in fact tightly intertwined in the quantum world. 
We shall prove Theorem 1.3 first. The proof will require a discussion of the convergence of the relevant Rogers-Fine series at roots of unity, the calculation of asymptotic expansions in terms of values of $L$-functions, and the mock modularity of two families of $q$-hypergeometric series. We shall use results of Lawrence and Zagier [31] and work of Bringmann and the second two authors [17] in this regard. These results are recalled in Section 2.1, and the proof of Theorem 1.3 is then given in Section 2.2.

To prove Theorem 1.2, which in turn implies Theorem 1.1, we make use of a beautiful identity of Choi [20] and Ramanujan (see [4, Entry 3.4.7]). This identity reduces the proof of Theorem 1.2 to the claim, upon appropriate specialization of variables, that a certain mixed mock modular form is asymptotic to a suitable multiple of the modular crank function. To establish this claim, we carry out a careful analysis of the asymptotic properties of modular Klein functions, certain Lerch-type series, and Mordell integrals. This is done in Section 3.

\section{Proof of Theorem 1.3}

Here, we prove Theorem 1.3 after first proving a lemma about the convergence of relevant $q$-hypergeometric series at roots of unity, and after recalling important results of Lawrence and Zagier [31] and Bringmann and the second two authors [17].

2.1. Preliminaries for the proof of Theorem 1.3. We begin with a simple lemma.

LeMMA 2.1. Let $0<a<b$ be coprime integers, where $b$ is even, and let $Q_{a, b}$ be as in the statement of Theorem 1.3. If $x=h / k \in Q_{a, b}$, then $G(a, b ;-x)$ and $H(a, b ; 1 / 2 x)$ converge. Moreover, they are explicitly given by the finite sums

$$
\begin{aligned}
G\left(a, b ;-\frac{h}{k}\right) & =\zeta_{b^{2} k}^{-a^{2} h} \sum_{n=0}^{m} \frac{\left(-\zeta_{b k}^{-a h} ; \zeta_{k}^{-h}\right)_{n}\left(-\zeta_{b k}^{-a h}\right)^{n}}{\left(\zeta_{b k}^{-a h} ; \zeta_{k}^{-h}\right)_{n+1}}, \\
H\left(a, b ; \frac{k}{2 h}\right) & =\zeta_{16 h}^{k} \sum_{n=0}^{\ell} \frac{\left(\zeta_{b}^{-a} \zeta_{2 h}^{k} ; \zeta_{h}^{k}\right)_{n}\left(\zeta_{b}^{-a} \zeta_{2 h}^{k}\right)^{n}}{\left(\zeta_{b}^{-a} \zeta_{h}^{k} ; \zeta_{h}^{k}\right)_{n}}
\end{aligned}
$$

where the nonnegative integers $\ell$ and $m$ satisfy $b(2 \ell+1)=2 h$ and $a+b m=k$, respectively.

Proof. By the definition of $Q_{a, b}$, there is an integer $\ell \geq 0$ such that $(2 \ell+1)=$ $2 h / b$, and so $a / b \equiv(k(2 \ell+1) / 2 h)(\bmod 1)$. Using this, the fact that $k \geq a, k \equiv$ $a(\bmod b)$, and (1.9), we see that, for $n \geq \ell+1$, the numerator of the $n$th summand in the series defining $H(a, b ; k / 2 h)$ will be zero. Next, it is not difficult to show 
that there are no integers $s$ for which $a / b \equiv(k / h) s(\bmod 1)$. This implies that none of the denominators vanish. This proves the claim for $H(a, b ; k / 2 h)$. Next, by the definition of $Q_{a, b}$, there is a nonnegative integer $m$ for which $a+b m=k$. Arguing as above, one easily arrives at the conclusion for $G(a, b ;-h / k)$.

Theorem 1.3(2) concerns the quantum modularity of the functions $G(a, b ; x)$ and $H(a, b ; x)$. To derive this, we make use of earlier work of Bringmann and the second two authors. We summarize the required results from [17, Theorem 4.3 and Lemma 4.5] in the theorem below. These results involve the $q$-hypergeometric functions (These functions were also studied by Gordon and McIntosh in [27]. In [17, §4.1], the function $g(a, b ; z)$ is shown to be equal to what the authors call $H(a, 0, b ; z)$. We do not use this notation, to avoid confusion.)

$$
\begin{aligned}
g(a, b ; z) & :=\sum_{n=0}^{\infty} \frac{(-q ; q)_{n} q^{n(n+1) / 2}}{\left(q^{a / b} ; q\right)_{n+1}\left(q^{-a / b} q ; q\right)_{n+1}}, \\
h(a, b ; z) & :=\sum_{n=0}^{\infty} \frac{(-1)^{n} q^{n^{2}}\left(q ; q^{2}\right)_{n}}{\left(\zeta_{b}^{a} q^{2} ; q^{2}\right)_{n}\left(\zeta_{b}^{-a} q^{2} ; q^{2}\right)_{n}},
\end{aligned}
$$

and the important theta function

$$
T(a, b ; z):=i \sum_{n=-\infty}^{\infty}\left(n+\frac{1}{4}\right) \cosh \left(2 \pi i\left(n+\frac{1}{4}\right)\left(\frac{2 a}{b}-1\right)\right) q^{\left(n+\frac{1}{4}\right)^{2}} .
$$

Equations (2.7) and (2.8) will give explicit identities relating these functions to $G$ and $H$.

THEOREM 2.2 [17, Theorem 4.3 and Lemma 4.5]. Let $z \in \mathbb{H}$, and suppose that $0<a<b$ are coprime integers. We have that

$$
\begin{aligned}
q^{a / b(1-a / b)} g(a, b ; z)= & \sqrt{\frac{2 i}{z}} \frac{e^{\pi i / 8 z}}{4 \sin (\pi a / b)} h\left(a, b ; \frac{-1}{2 z}\right) \\
& +\int_{0}^{i \infty} \frac{(-i u)^{-3 / 2} T\left(a, b ;-\frac{1}{u}\right) d u}{\sqrt{-i(u+z)}} .
\end{aligned}
$$

Theorem 1.3(3) relates asymptotic expansions to values of $L$-functions. To obtain our result in this direction, we make use of the following proposition of Lawrence and Zagier [31].

Proposition 2.3 [31, p. 98]. Let $C: \mathbb{Z} \rightarrow \mathbb{C}$ be a periodic function with mean value 0 . Then the associated L-series $L(s, C)=\sum_{n=1}^{\infty} C(n) n^{-s}(\operatorname{Re}(s)>1)$ extends holomorphically to all of $\mathbb{C}$, and the function $\sum_{n=1}^{\infty} C(n) e^{-n^{2} t}(t>0)$ 
has the asymptotic expansion

$$
\sum_{n=1}^{\infty} C(n) e^{-n^{2} t} \sim \sum_{r=0}^{\infty} L(-2 r, C) \cdot \frac{(-t)^{r}}{r !}
$$

as $t \rightarrow 0^{+}$. The numbers $L(-r, C)$ are given explicitly by

$$
L(-r, C)=-\frac{M^{r}}{r+1} \sum_{n=1}^{M} C(n) B_{r+1}\left(\frac{n}{M}\right) \quad(r=0,1, \ldots),
$$

where $B_{k}(x)$ denotes the $k$ th Bernoulli polynomial and $M$ is any period of the function $C(n)$.

2.2. Proof of Theorem 1.3. We first prove (1). Let $\rho:=q^{-1}$, where $q=e^{2 \pi i z}$ and $z \in \mathbb{H}$. We compute

$$
\begin{aligned}
-\rho^{a / b-a^{2} / b^{2}} G(a, b ;-z) & =-\rho^{a / b} \sum_{n=0}^{\infty} \frac{\left(-\rho^{a / b} ; \rho\right)_{n}}{\left(\rho^{a / b} ; \rho\right)_{n+1}}\left(-\rho^{a / b}\right)^{n} \\
& =\sum_{n=0}^{\infty} \frac{\left(-q^{a / b} ; q\right)_{n}}{\left(q^{a / b} ; q\right)_{n+1}} q^{n(1-a / b)}=: G^{*}(a, b ; z),
\end{aligned}
$$

where we use the fact that

$$
(a ; \rho)_{n}=(-a)^{n} q^{-n(n-1) / 2}\left(a^{-1} ; q\right)_{n} .
$$

Thus, from (1.8) and (2.3), we see that the series defining $G(a, b ; z)$ is defined for $z \in \mathbb{H} \cup \mathbb{H}^{-}$. Similarly, using (2.4), it is not difficult to show that

$$
q^{\frac{1}{8}} H(a, b ;-z)=F\left(q^{-1} \zeta_{b}^{a}, \zeta_{b}^{a}, \zeta_{b}^{-a} ; q^{2}\right),
$$

and hence that $H(a, b ; z)$ is defined for $z \in \mathbb{H} \cup \mathbb{H}^{-}$. We justify the convergence of the series $F\left(q^{-1} \zeta_{b}^{a}, \zeta_{b}^{a}, \zeta_{b}^{-a} ; q^{2}\right)$ in (2.5) as follows. By considering the Rogers-Fine series $F(\alpha, \beta, t ; q)$ purely formally, we have the functional equation

$$
F\left(q^{-1} \zeta_{b}^{a}, \zeta_{b}^{a}, \zeta_{b}^{-a} ; q^{2}\right)=-\zeta_{b}^{a}\left(1-\frac{\zeta_{b}^{a}-q}{\zeta_{b}^{a}-1} F\left(q^{-1} \zeta_{b}^{a}, \zeta_{b}^{a}, \zeta_{b}^{-a} q^{2} ; q^{2}\right)\right)
$$

(See also [24, (2.4)].) Iterating this recurrence relationship, it follows that $F(\alpha, \beta, t ; q)$ converges for all $t \neq q^{-n}, n \in \mathbb{N}_{0}$. (See also [24, page 2].) This proves (1).

We now prove (2). We relate $G^{*}(a, b ; z)$ to the mock modular form $g(a, b ; z)$. The identity

$$
(1-t) F(\alpha, \beta ; t)=\sum_{n=0}^{\infty} \frac{\left(\frac{\beta}{\alpha} ; q\right)_{n}}{(\beta q ; q)_{n}(t q ; q)_{n}} \cdot(-\alpha t)^{n} q^{n(n+1) / 2}
$$


(see [24, (12.2)]) with $\alpha=-q^{a / b-1}, \beta=q^{a / b}, t=q^{1-a / b}$ shows that

$$
G^{*}(a, b ; z)=g(a, b ; z) \text {. }
$$

We use (2.6) again with $\alpha=q^{-1} \zeta_{b}^{a}, \beta=\zeta_{b}^{a}, t=\zeta_{b}^{-a}$, and $q \rightarrow q^{2}$, and find that

$$
h(a, b ; z)=\left(1-\zeta_{b}^{-a}\right) F\left(q^{-1} \zeta_{b}^{a}, \zeta_{b}^{a}, \zeta_{b}^{-a} ; q^{2}\right)=\left(1-\zeta_{b}^{-a}\right) q^{\frac{1}{8}} H(a, b ;-z),
$$

where the last equality in (2.8) follows from (2.5). The proof of part (2) now follows from part (1), (2.3), (2.5), (2.7), (2.8), and Theorem 2.2. While Theorem 2.2 gives a transformation law for $z \in \mathbb{H}$, we have continuation to $x \in Q_{a, b}$ by Lemma 2.1. As argued in [41] and [19], the integral appearing in (2) of Theorem 1.3 is real analytic.

We now prove part (3). The conclusion of part (3) follows from Proposition 2.3 once the hypotheses are confirmed for certain $L$-functions related to $G(a, b ; z)$ and $H(a, b ; z)$.

To this end, we let $c_{G}(n)$ and $c_{H}(n)$ be as defined in (1.12) and (1.13), respectively. Then, using (1.10) and (1.11), we have

$$
\begin{aligned}
G\left(a, b ; \frac{-h}{k}+\frac{i t}{2 \pi}\right) & =\sum_{n=0}^{\infty} c_{G}(n) e^{-n^{2} t / b^{2}}, \\
H\left(a, b ; \frac{k}{2 h}+\frac{i t}{2 \pi}\right) & =\sum_{n=0}^{\infty} c_{H}(n) e^{-n^{2} t / 8} .
\end{aligned}
$$

It is clear that $c_{G}$ is $2 k b^{2}$ periodic, and $c_{H}$ is $16 h$ periodic. To prove that $c_{G}$ and $c_{H}$ have mean value zero, we establish the following claim. Let $a$ and $b$ be positive integers satisfying $\operatorname{gcd}(a, b)=1$, with $b$ even. Let $h$ and $k$ be integers such that $h / k \in Q_{a, b}$. Then $c_{G}$ and $c_{H}$ have mean value zero with periods $2 k b^{2}$ and $16 h$, respectively. The truth of this claim, then combined with Proposition 2.3, gives part (3).

We begin with $c_{G}$. Because $\operatorname{gcd}(a, b)=1$, there is some integer $\bar{a}$ such that $a \bar{a} \equiv 1(\bmod b)$. We have

$$
\sum_{\left(\bmod 2 k b^{2}\right)} c_{G}(n)=\Sigma_{1}-\Sigma_{2}
$$

where

$$
\Sigma_{1}:=\sum_{\substack{n\left(\bmod 2 k b^{2}\right) \\ n \equiv a(\bmod 2 b)}} \zeta_{k b^{2}}^{-h n^{2}}, \quad \Sigma_{2}:=\sum_{\substack{n\left(\bmod 2 k b^{2}\right) \\ n \equiv a+b(\bmod 2 b)}} \zeta_{k b^{2}}^{-h n^{2}}
$$


We have

$$
\Sigma_{1}=\sum_{\substack{n\left(\bmod 2 k k^{2}\right) \\ n \equiv a(\bmod 2 b)}} \zeta_{k b^{2}}^{-h n^{2}}=\sum_{\substack{n=1-b k \bar{a} \\ n \equiv a(\bmod 2 b)}}^{2 k b^{2}-b k \bar{a}} \zeta_{k b^{2}}^{-h n^{2}}=\sum_{\substack{n=1 \\ n \equiv a+b k \bar{a}(\bmod 2 b)}}^{2 k b^{2}} \zeta_{k b^{2}}^{-h(n-b k \bar{a})^{2}}
$$

Now, by hypothesis, there exists some integer $s$ (namely, $s=2 \ell+1$ ) such that $h=b s / 2$. Thus,

$$
\zeta_{k b^{2}}^{-h(n-b k \bar{a})^{2}}=\zeta_{k b^{2}}^{-h n^{2}} \zeta_{b}^{2 h n \bar{a}}=\zeta_{k b^{2}}^{-h n^{2}} \zeta_{b}^{b s n \bar{a}}=\zeta_{k b^{2}}^{-h n^{2}} .
$$

There also is an integer $m$ for which $k=a+b m$, and an integer $v$ with $a \bar{a}=1+b v$, so that

$$
\begin{aligned}
a+b k \bar{a} & =a+b(a+b m) \bar{a}=a+a \bar{a} b+\bar{a} m b^{2} \\
& =a+(1+b v) b+\bar{a} m b^{2}=a+b+b^{2}(v+\bar{a} m) \equiv a+b(\bmod 2 b),
\end{aligned}
$$

where the last equivalence follows because $b$ is even. We use (2.10) and (2.11) to rewrite (2.9) as

$$
\sum_{\substack{n=1 \\ n \equiv a+b(\bmod 2 b) \\ 2 k b^{2}}} \zeta_{k b^{2}}^{-h n^{2}}
$$

which is precisely equal to $\Sigma_{2}$.

To prove that $c_{H}$ has mean value with the given period, we use that $\ell$ is the nonnegative integer such that $b(2 \ell+1)=2 h$, and write

$$
\sum_{n(\bmod 16 h)} c_{H}(n)=\sum_{\substack{n=1 \\ n \text { odd }}}^{8 b(2 \ell+1)} \zeta_{b}^{-a((n-1) / 2)} \zeta_{8 b(2 \ell+1)}^{k n^{2}}=\Sigma_{3}+\Sigma_{4}
$$

where

$$
\Sigma_{3}:=\sum_{\substack{n=1 \\ n \text { odd }}}^{4 b(2 \ell+1)} \zeta_{b}^{-a((n-1) / 2)} \zeta_{8 b(2 \ell+1)}^{k n^{2}}, \quad \Sigma_{4}:=\sum_{\substack{n=4 b(2 \ell+1)+1 \\ n \text { odd }}}^{8 b(2 \ell+1)} \zeta_{b}^{-a((n-1) / 2)} \zeta_{8 b(2 \ell+1)}^{k n^{2}} .
$$

By replacing $n$ by $N+4 b(2 \ell+1)$ in $\Sigma_{4}$ and summing on $N$, we find that $\Sigma_{4}=\Sigma_{3}$, and hence $\sum_{n(\bmod 16 h)} c_{H}(n)=2 \Sigma_{3}$. Next, we rewrite $\Sigma_{3}$ as $\Sigma_{31}+\Sigma_{32}$, where

$$
\Sigma_{31}:=\sum_{\substack{n=1 \\ n \text { odd }}}^{2 b(2 \ell+1)} \zeta_{b}^{-a((n-1) / 2)} \zeta_{8 b(2 \ell+1)}^{k n^{2}}, \quad \Sigma_{32}:=\sum_{\substack{n=2 b(2 \ell+1)+1 \\ n \text { odd }}}^{4 b(2 \ell+1)} \zeta_{b}^{-a((n-1) / 2)} \zeta_{8 b(2 \ell+1)}^{k n^{2}}
$$


We rewrite $\Sigma_{32}$ as

$$
\Sigma_{32}=\sum_{\substack{n=1 \\ n \text { odd }}}^{2 b(2 \ell+1)} \zeta_{b}^{-a((n-1) / 2+b(2 \ell+1))} \zeta_{8 b(2 \ell+1)}^{k(n+2 b(2 \ell+1))^{2}}=\sum_{\substack{n=1 \\ n \text { odd }}}^{2 b(2 \ell+1)} \zeta_{b}^{-a((n-1) / 2)} \zeta_{8 b(2 \ell+1)}^{k n^{2}} \zeta_{2}^{n k}
$$

Because $k \equiv a(\bmod b), b$ is even, and $\operatorname{gcd}(a, b)=1$, we must have that $k$ is odd. Thus, $\Sigma_{32}=-\Sigma_{31}$, and hence $c_{H}(n)$ has mean value zero with period $16 h$, as claimed.

\section{Proof of Theorem 1.2}

To prove Theorem 1.2, we will require some preliminary results pertaining to ordinary modular forms, modular units, mock modular forms, and Jacobi theta functions. We describe these results in the following subsection. We then conclude with the proof of Theorem 1.2.

\subsection{Preliminaries for the proof of Theorem 1.2.}

3.1.1. Special modular forms. A modular form we require is the Dedekind $\eta$-function, defined for $q=e^{2 \pi i z}, z \in \mathbb{H}$, by

$$
\eta(z):=q^{\frac{1}{24}} \prod_{n=1}^{\infty}\left(1-q^{n}\right)
$$

It is well known [36] that $\eta(z)$ is modular of weight $1 / 2$, and that it transforms under $\gamma=\left(\begin{array}{ll}a & b \\ c & d\end{array}\right) \in \mathrm{SL}_{2}(\mathbb{Z})$ by

$$
\eta(\gamma z)=\psi(\gamma)(c z+d)^{\frac{1}{2}} \eta(z)
$$

where $\psi(\gamma)$ is a 24 th root of unity.

We will also require the Klein forms $\mathfrak{t}_{(r, s)}(z)=\mathfrak{t}_{(r, s)}^{(N)}(z)$ defined for pairs $(r, s) \in$ $\mathbb{Z}^{2}$ with respect to a positive integer level $N$, such that $(r, s) \not \equiv(0,0)(\bmod N \times$ $N$ ). These functions are defined using the Weierstrass $\sigma$-function, and they were studied originally by Klein and Fricke. Here, we give some of their key properties as summarized in a more modern source [30]:

$$
\begin{gathered}
\mathfrak{t}_{(r, s)}(\gamma z)=(c z+d)^{-1} \mathfrak{t}_{(r, s) \gamma}(z), \quad \text { for all } \gamma=\left(\begin{array}{ll}
a & b \\
c & d
\end{array}\right) \in \mathrm{SL}_{2}(\mathbb{Z}), \quad(3.2) \\
\mathfrak{t}_{(r, s)}(z)=-\frac{\zeta_{2 N^{2}}^{s(r-N)}}{2 \pi i} q^{r(r-N) / 2 N^{2}}\left(1-\zeta_{N}^{s} q^{r / N}\right) \prod_{n=1}^{\infty} \frac{\left(1-\zeta_{N}^{s} q^{n+r / N}\right)\left(1-\zeta_{N}^{-s} q^{n-r / N}\right)}{\left(1-q^{n}\right)^{2}},
\end{gathered}
$$

where $q=e^{2 \pi i z}$, and $(r, s) \gamma$ denotes matrix multiplication. 
3.1.2. Mock Jacobi forms. In his celebrated Ph.D. thesis [45], Zwegers defined the Appell-Lerch sums for $q=e^{2 \pi i z}, z \in \mathbb{H}$, and $u, v \in \mathbb{C} \backslash(\mathbb{Z} z+\mathbb{Z})$ by

$$
\mu(u, v ; z):=\frac{e^{\pi i u}}{\vartheta(v ; z)} \sum_{n \in \mathbb{Z}} \frac{(-1)^{n} q^{n(n+1) / 2} e^{2 \pi i n v}}{1-e^{2 \pi i u} q^{n}} .
$$

Here, the Jacobi theta function is defined by

$$
\begin{aligned}
\vartheta(v ; z) & :=i \sum_{n \in \mathbb{Z}}(-1)^{n} q^{\frac{1}{2}\left(n+\frac{1}{2}\right)^{2}} e^{2 \pi i v\left(n+\frac{1}{2}\right)} \\
& =-i q^{\frac{1}{8}} e^{-\pi i v} \prod_{n=1}^{\infty}\left(1-q^{n}\right)\left(1-e^{2 \pi i v} q^{n-1}\right)\left(1-e^{-2 \pi i v} q^{n}\right) .
\end{aligned}
$$

The last equality in (3.4) is the well-known Jacobi product identity. The Jacobi form $\vartheta(v ; z)$ transforms as follows [36]:

$$
\begin{aligned}
& \vartheta(v+\alpha z+\beta ; z)=(-1)^{\alpha+\beta} q^{-\alpha^{2} / 2} e^{-2 \pi i \alpha v} \vartheta(v ; z), \\
& \vartheta\left(\frac{v}{c z+d} ; \gamma z\right)=\rho(\gamma)(c z+d)^{1 / 2} e^{\pi i c v^{2} /(c z+d)} \vartheta(v ; z),
\end{aligned}
$$

for all $\alpha, \beta \in \mathbb{Z}$ and $\gamma=\left(\begin{array}{ll}a & b \\ c & d\end{array}\right) \in \mathrm{SL}_{2}(\mathbb{Z})$, where $\rho(\gamma)=(\psi(\gamma))^{3}$ is an eighth root of unity.

A result for the mock Jacobi forms $\mu(u, v ; z)$ that we will make use of is the following beautiful and important identity of Choi [20].

THEOREM 3.1 (Choi [20]). Let $q=e^{2 \pi i z}$, where $z \in \mathbb{H}$. For suitable complex numbers $\alpha=e^{2 \pi i u}$ and $\beta=e^{2 \pi i v}$, we have

$$
\begin{aligned}
& \sum_{n=0}^{\infty} \frac{(\alpha \beta)^{n} q^{n^{2}}}{(\alpha q ; q)_{n}(\beta q ; q)_{n}}+\sum_{n=1}^{\infty} q^{n}\left(\alpha^{-1} ; q\right)_{n}\left(\beta^{-1} ; q\right)_{n} \\
& \quad=i q^{\frac{1}{8}}(1-\alpha)\left(\beta \alpha^{-1}\right)^{\frac{1}{2}}\left(q \alpha^{-1} ; q\right)_{\infty}\left(\beta^{-1} ; q\right)_{\infty} \mu(u, v ; z) .
\end{aligned}
$$

REMARK. We note that Theorem 3.1 can be obtained from Entry 3.4.7 of Ramanujan's 'Lost Notebook' (see [4, p. 67]).

REMARK. We point out that the left-hand side of the displayed identity in Theorem 3.1 may also be rewritten as $\sum_{n=-\infty}^{\infty}(\alpha \beta)^{n} q^{n^{2}} /(\alpha q ; q)_{n}(\beta q ; q)_{n}$; however, the relevant expression here is the one given in Theorem 3.1.

To make use of Theorem 3.1, we shall require the modular transformation properties of $\mu(u, v ; z)$. Multivariable generalizations of the function $A(u, v ; z):=\vartheta(v ; z) \mu(u, v ; z)$ were studied by Zwegers [46] and the first author 
and Bringmann [12]. A 'completion' of this function is defined by Zwegers as

$$
\widehat{A}(u, v ; z):=A(u, v ; z)+\frac{i}{2} \vartheta(v ; z) R(u-v ; z),
$$

where

$$
\begin{aligned}
R(v ; z):= & \sum_{n \in \mathbb{Z}}\left\{\operatorname{sgn}\left(n+\frac{1}{2}\right)-E\left(\left(n+\frac{1}{2}+\frac{\operatorname{Im}(v)}{\operatorname{Im}(z)}\right) \sqrt{2 \cdot \operatorname{Im}(z)}\right)\right\} \\
& \times(-1)^{n} q^{-\frac{1}{2}\left(n+\frac{1}{2}\right)^{2}} e^{-2 \pi i v\left(n+\frac{1}{2}\right)},
\end{aligned}
$$

and for $w \in \mathbb{C}$ we have

$$
E(w):=2 \int_{0}^{w} e^{-\pi u^{2}} d u .
$$

The functions $R(v ; z)$ transform as follows [45] under the generators of $\mathrm{SL}_{2}(\mathbb{Z})$ :

$$
\begin{gathered}
R(v ; z+1)=e^{-\pi i / 4} R(v ; z), \\
\frac{1}{\sqrt{-i z}} e^{\pi i v^{2} / z} R\left(\frac{v}{z} ;-\frac{1}{z}\right)+R(v ; z)=h(v ; z),
\end{gathered}
$$

where the Mordell integral $h(v ; z)$ is defined by

$$
h(v ; z):=\int_{-\infty}^{\infty} \frac{e^{\pi i z u^{2}-2 \pi v u}}{\cosh (\pi u)} d u .
$$

Using the transformation properties of the functions $\mu$ (see [45]) and $\vartheta$ (see (3.5) and (3.6)), we have, for integers $m, n, r, s$, and $\gamma=\left(\begin{array}{ll}a & b \\ c & d\end{array}\right) \in \mathrm{SL}_{2}(\mathbb{Z})$, that

$$
\begin{gathered}
\widehat{A}(u+m z+n, v+r z+s ; z)=(-1)^{m+n} e^{2 \pi i u(m-r)} e^{-2 \pi i v m} q^{m^{2} / 2-m r} \widehat{A}(u, v ; z), \\
\widehat{A}\left(\frac{u}{c z+d}, \frac{v}{c z+d} ; \gamma z\right)=(c z+d) e^{\pi i c\left(-u^{2}+2 u v\right) /(c z+d)} \widehat{A}(u, v ; z)
\end{gathered}
$$

3.2. Proof of Theorem 1.2. To prove Theorem 1.2, we will first make use of Choi's identity in Theorem 3.1 with $\alpha=\zeta_{b}^{-a}$ and $\beta=\zeta_{b}^{a}$ (hence $u=-a / b$, $v=a / b)$, and $q$ replaced by $e^{(2 \pi i / k)(h+i z)}$. We define

$$
m(a, b ; u):=i e^{\pi i u / 4}\left(1-\zeta_{b}^{-a}\right) \zeta_{b}^{a}\left(\zeta_{b}^{a} e^{2 \pi i u} ; e^{2 \pi i u}\right)_{\infty}\left(\zeta_{b}^{-a} ; e^{2 \pi i u}\right)_{\infty}
$$


To prove Theorem 1.2, noting that the function $U\left(\zeta_{b}^{a} ; \zeta_{k}^{h}\right)$ is a finite convergent sum when $b \mid k$, by the argument above, it thus suffices to prove that, upon appropriate specialization of variables, the mixed mock modular form $m \cdot \mu$ is asymptotic to a suitable multiple of the modular crank-generating function $C$.

To be precise, let $b \mid k, \operatorname{gcd}(a, b)=1, \operatorname{gcd}(h, k)=1$, where $a, b, h, k$ are positive integers. By comparing the asymptotics in Theorem 3.2 and Proposition 3.3 below, we immediately find that, as $z \rightarrow 0^{+}$,

$$
m\left(a, b ; \frac{1}{k}(h+i z)\right) \mu\left(-\frac{a}{b}, \frac{a}{b} ; \frac{1}{k}(h+i z)\right) \sim \zeta_{b^{2}}^{-a^{2} h^{\prime} k} C\left(\zeta_{b}^{a} ; \frac{1}{k}(h+i z)\right) .
$$

The error terms in Theorem 3.2 and Proposition 3.3 complete the proof.

REMARK. Above, and in what follows, we let $z \in \mathbb{R}^{+}$, and let $z \rightarrow 0^{+}$. This corresponds to $q=e^{(2 \pi i / k)(h+i z)} \rightarrow \zeta_{k}^{h}$ from within the unit disk, as described in the statements of Theorems 1.1 and 1.2.

Therefore, it remains to obtain these two separate asymptotic results for the crank function and the mixed mock modular form in question. To describe this, we let

$$
q:=e^{(2 \pi i / k)(h+i z)}, \quad q_{1}:=e^{(2 \pi i / k)\left(h^{\prime}+i / z\right)} .
$$

For the mixed mock modular $m \cdot \mu$, we obtain the following asymptotics.

THEOREM 3.2. Let $b \mid k, \operatorname{gcd}(a, b)=1, \operatorname{gcd}(h, k)=1$, where $a, b, h, k$ are positive integers, and let $b^{\prime}$ and $h^{\prime}$ be positive integers such that $b b^{\prime}=k$ and $h h^{\prime} \equiv-1(\bmod k)$. For $z \in \mathbb{R}^{+}$, as $z \rightarrow 0^{+}$, we have that

$$
\begin{aligned}
& m\left(a, b ; \frac{1}{k}(h+i z)\right) \mu\left(-\frac{a}{b}, \frac{a}{b} ; \frac{1}{k}(h+i z)\right) \\
& \quad=\left(\frac{i}{z}\right)^{\frac{1}{2}}(\psi(\gamma))^{-1} q^{\frac{1}{24}} q_{1}^{-\frac{1}{24}}(-1)^{a b^{\prime}} \zeta_{2 b}^{a h^{\prime}-a} \zeta_{2 b^{2}}^{-3 a^{2} k h^{\prime}} \frac{\zeta_{b}^{a}-1}{1-\zeta_{b}^{a h^{\prime}}}\left(1+O\left(q_{1}^{\alpha}\right)\right),
\end{aligned}
$$

for some $\alpha>1 / 24$.

Combining Theorem 3.2 with the following asymptotics for the modular crank function then gives (3.13), which in turn then implies Theorem 1.2 as argued above. The reader should note the multiplicative constant in front of $C\left(\zeta_{b}^{a} ; q\right)$ in the statement of Theorem 1.2.

Proposition 3.3. Let $b \mid k, \operatorname{gcd}(a, b)=1, \operatorname{gcd}(h, k)=1$, where $a, b, h, k$ are positive integers, and let $b^{\prime}$ and $h^{\prime}$ be positive integers such that $b b^{\prime}=k$ 
and $h h^{\prime} \equiv-1(\bmod k)$. For $z \in \mathbb{R}^{+}$, as $z \rightarrow 0^{+}$, we have that

$$
\begin{aligned}
C\left(\zeta_{b}^{a} ; \frac{1}{k}(h+i z)\right)=\left(\frac{i}{z}\right)^{\frac{1}{2}}(\psi(\gamma))^{-1} q^{\frac{1}{24}} q_{1}^{-\frac{1}{24}}(-1)^{a b^{\prime}} \zeta_{2 b}^{a h^{\prime}-a} \zeta_{2 b^{2}}^{-a^{2} k h^{\prime}} \\
\times \frac{\zeta_{b}^{a}-1}{1-\zeta_{b}^{a h^{\prime}}}\left(1+O\left(q_{1}^{\beta}\right)\right),
\end{aligned}
$$

for some $\beta>1 / 24$.

\subsection{Proof of Theorem 3.2. We define}

$$
Z:=\frac{1}{k}(h+i z), \quad Z^{\prime}:=\frac{1}{k}\left(h^{\prime}+\frac{i}{z}\right), \quad \gamma:=\left(\begin{array}{cc}
h & -\left(\frac{h h^{\prime}+1}{k}\right) \\
k & -h^{\prime}
\end{array}\right) .
$$

Thus, we have that $\gamma Z^{\prime}=Z$, and, because $z \in \mathbb{R}^{+}$, we have that $Z \in \mathbb{H}, Z^{\prime} \in \mathbb{H}$. Using (3.4), (3.5), and the fact that $\vartheta(v ; z)$ is an odd function with respect to $v$, we find that

$$
m(a, b ; Z)=q^{\frac{1}{24}} \zeta_{2 b}^{-a}\left(\zeta_{b}^{a}-1\right) \frac{\vartheta\left(\frac{a}{b} ; Z\right)}{\eta(Z)}
$$

(where we recall that $q=e^{2 \pi i Z}$ ). Using this, we find that

$$
\begin{aligned}
m(a, b ; Z) \mu\left(-\frac{a}{b}, \frac{a}{b} ; Z\right) & :=m(a, b ; Z) \frac{A\left(-\frac{a}{b}, \frac{a}{b} ; Z\right)}{\vartheta\left(\frac{a}{b} ; Z\right)} \\
& =\frac{q^{\frac{1}{24}} \zeta_{2 b}^{-a}\left(\zeta_{b}^{a}-1\right)}{\eta(Z)} A\left(-\frac{a}{b}, \frac{a}{b} ; Z\right),
\end{aligned}
$$

where we recall that

$$
A(u, v ; Z):=e^{\pi i u} \sum_{n \in \mathbb{Z}} \frac{(-1)^{n} q^{(n(n+1) / 2) e^{2 \pi i n v}}}{1-e(u) q^{n}} .
$$

Using the fact that $Z=\gamma Z^{\prime}$, together with (3.11), we find that the completed function $\widehat{A}$ (defined in (3.7)) satisfies

$$
\widehat{A}\left(-\frac{a}{b}, \frac{a}{b} ; Z\right)=\widehat{A}\left(-\frac{a}{b}, \frac{a}{b} ; \gamma Z^{\prime}\right)=\left(\frac{i}{z}\right) e^{-\left(3 \pi i k a^{2} / b^{2}\right) \cdot(i / z)} \widehat{A}\left(-\frac{a i}{b z}, \frac{a i}{b z} ; Z^{\prime}\right) .
$$


Next, by hypothesis, there exists some positive integer $b^{\prime}$ such that $b b^{\prime}=k$. Thus, we rewrite

$$
\begin{aligned}
\left(\begin{array}{c}
i \\
z
\end{array}\right) e^{-\left(3 \pi i k a^{2} / b^{2}\right) \cdot(i / z)} \widehat{A}\left(-\frac{a i}{b z}, \frac{a i}{b z} ; Z^{\prime}\right) \\
=\left(\begin{array}{c}
i \\
z
\end{array}\right) e^{-\left(3 \pi i k a^{2} / b^{2}\right) \cdot(i / z)} \widehat{A}\left(\frac{a b^{\prime} h^{\prime}}{k}-a b^{\prime} Z^{\prime},-\frac{a b^{\prime} h^{\prime}}{k}+a b^{\prime} Z^{\prime} ; Z^{\prime}\right) \\
=\left(\begin{array}{c}
i \\
z
\end{array}\right)(-1)^{a b^{\prime}} \zeta_{2 b^{2}}^{-3 a^{2} k h^{\prime}} \widehat{A}\left(\frac{a h^{\prime}}{b},-\frac{a h^{\prime}}{b} ; Z^{\prime}\right),
\end{aligned}
$$

where we have made use of (3.10).

From (3.15) and (3.16), also using the definition (3.7), we rewrite

$$
\begin{aligned}
m(a, b ; Z) & \mu\left(-\frac{a}{b}, \frac{a}{b} ; Z\right) \\
= & \frac{q^{\frac{1}{24}} \zeta_{2 b}^{-a}\left(\zeta_{b}^{a}-1\right)}{\eta(Z)}\left(\frac{i}{z}\right)(-1)^{a b^{\prime}} \zeta_{2 b^{2}}^{-3 a^{2} k h^{\prime}} A\left(\frac{a h^{\prime}}{b},-\frac{a h^{\prime}}{b} ; Z^{\prime}\right) \\
+ & \frac{q^{\frac{1}{24}} \zeta_{2 b}^{-a}\left(\zeta_{b}^{a}-1\right)}{\eta(Z)}\left(\frac{i}{z}\right)(-1)^{a b^{\prime}} \zeta_{2 b^{2}}^{-3 a^{2} k h^{\prime}}\left(\frac{i}{2}\right) \\
& \times R\left(\frac{2 a h^{\prime}}{b} ; Z^{\prime}\right) \vartheta\left(-\frac{a h^{\prime}}{b} ; Z^{\prime}\right) \\
& -\frac{q^{\frac{1}{24}} \zeta_{2 b}^{-a}\left(\zeta_{b}^{a}-1\right)}{\eta(Z)}\left(\frac{i}{2}\right) R\left(-\frac{2 a}{b} ; Z\right) \vartheta\left(\frac{a}{b} ; \gamma Z^{\prime}\right)
\end{aligned}
$$

LEMMA 3.4. With hypotheses as above, we have that

$$
\begin{aligned}
R\left(-\frac{2 a}{b} ; Z\right)=\frac{e^{-\pi i h k / 4+\pi i(k-1)(-2 a / b+1 / 2)}(-1)^{(k-1)(h-1) / 2}}{\sqrt{k z}} & \\
& \times \sum_{\ell=0}^{k-1} e^{-(\pi i h / k)(\ell-(k-1) / 2)^{2}} \zeta_{b}^{2 a \ell}(-1)^{\ell(h+1)} \\
& \times\left(-R\left(\frac{\ell-\frac{k-1}{2}}{k} ; \frac{i}{k z}\right)+h\left(\frac{\ell-\frac{k-1}{2}}{k} ; \frac{i}{k z}\right)\right) .
\end{aligned}
$$

Proof of Lemma 3.4. We first apply a dissection result for the functions $R(v ; z)$ proved by the first author and Bringmann in [12, Proposition 2.3]. A direct application to the function $R(-2 a / b ;(h+i z) / k)$ results in a sum on $\ell$, where $\ell$ 
ranges from 0 to $k-1$, of terms involving the function

$$
R\left(-\frac{2 a k}{b}+\ell h-\frac{(k-1)(h-1)}{2}+i z\left(\ell-\frac{k-1}{2}\right) ; k h+i k z\right) .
$$

Now, because $h$ and $k$ are relatively prime, at least one of $h$ and $k$ must be odd. Moreover, we recall that $b \mid k$. Together, these facts imply that

$$
-\frac{2 a k}{b}+\ell h-\frac{(k-1)(h-1)}{2} \in \mathbb{Z} .
$$

Next, we use that $R(v+1 ; z)=-R(v)$ [45], as well as (3.8) and (3.9). A short calculation gives the desired result.

Resuming the proof of Theorem 3.2, we apply Lemma 3.4 to (3.17) and find that

$$
m(a, b ; Z) \mu\left(-\frac{a}{b}, \frac{a}{b} ; Z\right)=\Sigma_{1}+\Sigma_{2}+\Sigma_{3}+\Sigma_{4},
$$

where

$$
\begin{aligned}
\Sigma_{1}:= & \frac{q^{1 / 24} \zeta_{2 b}^{-a}\left(\zeta_{b}^{a}-1\right)}{\eta(Z)}\left(\frac{i}{z}\right)(-1)^{a b^{\prime}} \zeta_{2 b^{2}}^{-3 a^{2} k h^{\prime}} A\left(\frac{a h^{\prime}}{b},-\frac{a h^{\prime}}{b} ; Z^{\prime}\right), \\
\Sigma_{2}:= & \frac{q^{1 / 24} \zeta_{2 b}^{-a}\left(\zeta_{b}^{a}-1\right)}{\eta(Z)}\left(\frac{i}{z}\right)(-1)^{a b^{\prime}} \zeta_{2 b^{2}}^{-3 a^{2} k h^{\prime}}\left(\frac{i}{2}\right) R\left(\frac{2 a h^{\prime}}{b} ; Z^{\prime}\right) \vartheta\left(-\frac{a h^{\prime}}{b} ; Z^{\prime}\right), \\
\Sigma_{3}:= & \frac{q^{1 / 24} \zeta_{2 b}^{-a}\left(\zeta_{b}^{a}-1\right)}{\eta(Z)}\left(\frac{i}{2}\right) \vartheta\left(\frac{a}{b} ; \gamma Z^{\prime}\right) \\
& \times \frac{e^{-\pi i h k / 4+\pi i(k-1)(-2 a / b+1 / 2)}(-1)^{(k-1)(h-1) / 2}}{\sqrt{k z}} \\
& \times \sum_{\ell=0}^{k-1} e^{-(\pi i h / k)(\ell-(k-1) / 2)^{2}} \zeta_{b}^{2 a \ell}(-1)^{\ell(h+1)} R\left(\frac{\ell-\frac{k-1}{2}}{k} ; \frac{i}{k z}\right), \\
\Sigma_{4}:=- & \frac{q^{1 / 24} \zeta_{2 b}^{-a}\left(\zeta_{b}^{a}-1\right)}{\eta(Z)}\left(\frac{i}{2}\right) \vartheta\left(\frac{a}{b} ; \gamma Z^{\prime}\right) \\
& \times \frac{e^{-\pi i h k / 4+\pi i(k-1)(-2 a / b+1 / 2)}(-1)^{(k-1)(h-1) / 2}}{\sqrt{k z}} \\
& \times \sum_{\ell=0}^{k-1} e^{-(\pi i h / k)(\ell-(k-1) / 2)^{2}} \zeta_{b}^{2 a \ell}(-1)^{\ell(h+1)} h\left(\frac{\ell-\frac{k-1}{2}}{k} ; \frac{i}{k z}\right) .
\end{aligned}
$$


LEMMA 3.5. With hypotheses as above, we have that $\Sigma_{2}+\Sigma_{3}=0$.

Proof. We prove this by first using the fact that

$$
\vartheta\left(\frac{a}{b} ; \gamma Z^{\prime}\right)=\rho(\gamma)\left(\frac{i}{z}\right)^{\frac{1}{2}} \zeta_{2 b^{2}}^{h^{\prime} a^{2} k}(-1)^{a b^{\prime}} \vartheta\left(-\frac{a h^{\prime}}{b} ; Z^{\prime}\right),
$$

where $\rho(\gamma)$ is an eighth root of unity. This follows after applying (3.5) and (3.6), using that $a i / b z=a b^{\prime} Z^{\prime}-a h^{\prime} / b$. Using (3.19), it suffices to show that

$$
i \zeta_{2 b^{2}}^{-3 a^{2} k h} R\left(\frac{2 a h^{\prime}}{b} ; Z^{\prime}\right)
$$

equals

$$
\begin{aligned}
& \sqrt{\frac{i}{k}} e^{-\pi i h k / 4} e^{\pi i(k-1)(-2 a / b+1 / 2)}(-1)^{(k-1)(h-1) / 2} \rho(\gamma) \zeta_{2 b^{2}}^{h^{\prime} a^{2} k} \\
& \quad \times \sum_{\ell=0}^{k-1} e^{-(\pi i h / k)(\ell-(k-1) / 2)^{2}} \zeta_{b}^{2 a \ell}(-1)^{\ell(h+1)} R\left(\frac{\ell-\frac{k-1}{2}}{k} ; \frac{i}{k z}\right) .
\end{aligned}
$$

This follows in a similar manner to the argument used by the third author and Bringmann and Mahlburg to prove Proposition 2.7 in [14]. Namely, all other terms in (3.18) are meromorphic; thus it suffices to show that each term in (3.20) and (3.21) has a Fourier expansion of the form

$$
\sum_{n \in \mathbb{Q} \backslash\{0\}} a(n) \Gamma\left(\frac{1}{2} ; 4 \pi|n| y\right) q^{-n},
$$

where $\Gamma(\alpha ; x):=\int_{x}^{\infty} e^{-t} t^{\alpha-1} d t$. This follows according to the argument given [14].

LEMMA 3.6. With hypotheses as above, we have that

$$
\Sigma_{4}=q^{\frac{1}{24}} q_{1}^{\frac{1}{12}} \kappa\left(1+O\left(q_{1}^{\alpha}\right)\right)
$$

for some constant $\kappa$, and some $\alpha>0$ as $z \rightarrow 0^{+}$.

Proof. We first note, using (3.1) and the definition of $\eta(z)$, that

$$
\eta^{-1}\left(\gamma Z^{\prime}\right)=\left(\frac{i}{z}\right)^{-\frac{1}{2}}(\psi(\gamma))^{-1} \eta^{-1}\left(Z^{\prime}\right)=\left(\frac{i}{z}\right)^{-\frac{1}{2}} q_{1}^{-\frac{1}{24}}(\psi(\gamma))^{-1}\left(1+O\left(q_{1}^{\alpha_{2}}\right)\right)
$$

for some $\alpha_{2}>1 / 24$ as $z \rightarrow 0^{+}$. Next, let $c \in \mathbb{Q}$. Then, by definition,

$$
h\left(c ; \frac{i}{k z}\right)=\int_{-\infty}^{\infty} \frac{e^{-\pi x^{2} / k z-2 \pi c x}}{\cosh (\pi x)} d x .
$$


We have that $e^{-2 \pi c x} / \cosh (\pi x)=O(1)$ on $\mathbb{R}$, so

$$
h\left(c ; \frac{i}{k z}\right)=O\left(\int_{-\infty}^{\infty} e^{-\pi x^{2} / k z} d x\right)=O(\sqrt{z}),
$$

where we have used the fact that $z \in \mathbb{R}^{+}$. The proof of Lemma 3.6 now follows after a short calculation from this fact, the transformations (3.19) and (3.22), and from the series expansion for $\vartheta\left(x ; Z^{\prime}\right)$ for $x \in \mathbb{Q}$ given in (3.4).

LEMMA 3.7. With hypotheses as above, we have that

$$
A\left(\frac{a h^{\prime}}{b},-\frac{a h^{\prime}}{b} ; Z^{\prime}\right)=\frac{\zeta_{2 b}^{a h^{\prime}}}{1-\zeta_{b}^{a h^{\prime}}}\left(1+O\left(q_{1}^{\alpha_{1}}\right)\right)
$$

for some $\alpha_{1}>0$ as $z \rightarrow 0^{+}$.

Proof. This follows easily using the definition of $A(u, v ; z)$.

Theorem 3.2 now follows after combining (3.18), Lemma 3.5, and Lemma 3.6, noting that $\Sigma_{4} \rightarrow 0$ as $z \rightarrow 0^{+}$, as well as Lemma 3.7, and (3.22) to obtain

$$
\begin{aligned}
m(a, b ; Z) \mu\left(-\frac{a}{b}, \frac{a}{b} ; Z\right)=\left(\frac{i}{z}\right)^{\frac{1}{2}}(\psi(\gamma))^{-1} q^{\frac{1}{24}} q_{1}^{-\frac{1}{24}}(-1)^{a b^{\prime}} \zeta_{2 b}^{a h^{\prime}-a} \zeta_{2 b^{2}}^{-3 a^{2} k h^{\prime}} \\
\times \frac{\zeta_{b}^{a}-1}{1-\zeta_{b}^{a h^{\prime}}}\left(1+O\left(q_{1}^{\alpha_{3}}\right)\right)
\end{aligned}
$$

for some $\alpha_{3}>1 / 24$ as $z \rightarrow 0^{+}$.

3.4. Proof of Proposition 3.3. We use the product expansion for the Klein forms $\mathfrak{t}_{(r, s)}(z)=\mathfrak{t}_{(r, s)}^{(b)}(z)$ with respect to the modulus $b$ given in (3.3) and the definition of $\eta(z)$ to rewrite the crank-generating function as

$$
C\left(\zeta_{b}^{-a} ; Z\right)=-\frac{q^{\frac{1}{24}} \zeta_{2 b}^{-a}\left(1-\zeta_{b}^{a}\right)}{(2 \pi i) \eta(Z) \mathfrak{t}_{(0, a)}(Z)}=-\frac{q^{\frac{1}{24}} \zeta_{2 b}^{-a}\left(1-\zeta_{b}^{a}\right)}{(2 \pi i) \eta\left(\gamma Z^{\prime}\right) \mathfrak{t}_{(0, a)}\left(\gamma Z^{\prime}\right)},
$$

where we again use the fact that $Z=\gamma Z^{\prime}$ to obtain the last equality above. Next, we apply (3.2) and (3.22) to (3.24), and find that

$$
C\left(\zeta_{b}^{-a} ; Z\right)=-\frac{q^{\frac{1}{24}} q_{1}^{-\frac{1}{24}} \zeta_{2 b}^{-a}\left(1-\zeta_{b}^{a}\right)}{(2 \pi i) \mathfrak{t}_{\left(a k,-a h^{\prime}\right)}\left(Z^{\prime}\right)}\left(\frac{i}{z}\right)^{\frac{1}{2}}(\psi(\gamma))^{-1}\left(1+O\left(q_{1}^{\alpha_{2}}\right)\right)
$$


for some $\alpha_{2}>1 / 24$ as $z \rightarrow 0^{+}$. We next use (3.3)-(3.6) to rewrite

$$
\begin{aligned}
\mathfrak{t}_{\left(a k,-a h^{\prime}\right)}\left(Z^{\prime}\right) & =-\frac{i \zeta_{2 b^{2}}^{-a^{2} h^{\prime} k} q_{1}^{a^{2} k^{2} / 2 b^{2}} \vartheta\left(-\frac{a h^{\prime}}{b}+\frac{a}{b}\left(h^{\prime}+\frac{i}{z}\right) ; Z^{\prime}\right)}{(2 \pi i) \eta^{3}\left(Z^{\prime}\right)} \\
& =(-1)^{a b^{\prime}+1} \frac{i \zeta_{2 b^{2}}^{a^{2} h^{\prime} k} \vartheta\left(-\frac{a h^{\prime}}{b} ; Z^{\prime}\right)}{(2 \pi i) \eta^{3}\left(Z^{\prime}\right)} \\
& =(-1)^{a b^{\prime}} \frac{\zeta_{2 b^{2}}^{a^{2} h^{\prime} k}\left(\zeta_{2 b}^{-a h^{\prime}}-\zeta_{2 b}^{a h^{\prime}}\right)\left(1+O\left(q_{1}^{\alpha_{3}}\right)\right)}{(2 \pi i)},
\end{aligned}
$$

for some $\alpha_{3}>0$ as $z \rightarrow 0^{+}$. Thus, from (3.25) and (3.26), we have that $C\left(\zeta_{b}^{-a} ; Z\right)=\left(\frac{i}{z}\right)^{\frac{1}{2}} \psi(\gamma)^{-1} q^{\frac{1}{24}} q_{1}^{\frac{-1}{24}}(-1)^{a b^{\prime}+1} \zeta_{2 b}^{-a} \zeta_{2 b^{2}}^{-a^{2} h^{\prime} k} \frac{1-\zeta_{b}^{a}}{\zeta_{2 b}^{-a h^{\prime}}-\zeta_{2 b}^{a h^{\prime}}}\left(1+O\left(q_{1}^{\beta}\right)\right)$ for some $\beta>1 / 24$ as $z \rightarrow 0^{+}$. After some simplification, we obtain the proposition.

\section{Acknowledgements}

The authors thank Robert Lemke Oliver for computing the numerical examples in this paper, and they thank the referees, George Andrews, Bruce Berndt, Kathrin Bringmann, Jeremy Lovejoy, and Wadim Zudilin for helpful conversations. The authors thank the NSF and the Asa Griggs Candler Fund for their generous support.

\section{References}

[1] G. E. Andrews, 'On the theorems of Watson and Dragonette for Ramanujan's mock theta functions', Amer. J. Math. 88 (1966), 454-490.

[2] G. E. Andrews, 'Concave and convex compositions', Ramanujan J., to appear.

[3] G. E. Andrews and B. C. Berndt, 'Your hit parade - the top ten most fascinating formulas from Ramanujan's lost notebook', Notices Amer. Math. Soc. 55 (2008), 18-30.

[4] G. E. Andrews and B. C. Berndt, Ramanujan's Lost Notebook. Part II (Springer, New York, 2009).

[5] G. E. Andrews and F. Garvan, 'Dyson's crank of a partition', Bull. Amer. Math. Soc. (N.S.) 18 (1988), 167-171.

[6] G. E. Andrews, K. Ono and J. Urroz, ' $q$-identities and values of certain $L$-functions', Duke Math. J. 108 (2001), 395-419.

[7] G. E. Andrews, R. C. Rhoades and S. Zwegers, 'Modularity of the concave composition generating function', Algebra Number Theory, (2013), accepted for publication.

[8] A. O. L. Atkin and H. P. F. Swinnerton-Dyer, 'Some properties of partitions', Proc. Lond. Math. Soc. 4 (1954), 84-106. 
[9] J. Bajpai, S. Kimport, J. Liang, D. Ma and J. Ricci, 'Bilateral series and Ramanujan's radial limits', Proc. Amer. Math. Soc., (2013), accepted for publication.

[10] B. C. Berndt, 'Ramanujan, his lost notebook, its importance', preprint.

[11] B. C. Berndt and R. A. Rankin, Ramanujan: Letters and Commentary (American Mathematical Society, Providence, 1995).

[12] K. Bringmann and A. Folsom, 'On the asymptotic behavior of Kac-Wakimoto characters', Proc. Amer. Math. Soc. 141(5) (2013), 1567-1576.

[13] K. Bringmann, A. Folsom and R. C. Rhoades, 'Partial and mock theta functions as $q$-hypergeometric series', in Ramanujan's 125th Anniversary Special Volume, Ramanujan J. 29(1-3) (2012), 295-310 (special issue).

[14] K. Bringmann, K. Mahlburg and R. C. Rhoades, 'Taylor coefficients of mock-Jacobi forms and moments of partition statistics', Math. Proc. Cambridge Philos. Soc., to appear.

[15] K. Bringmann and K. Ono, 'The $f(q)$ mock theta function conjecture and partition ranks', Invent. Math. 165 (2006), 243-266.

[16] K. Bringmann and K. Ono, 'Dyson's ranks and Maass forms', Ann. of Math. 171 (2010), 419-449.

[17] K. Bringmann, K. Ono and R. Rhoades, 'Eulerian series as modular forms', J. Amer. Math. Soc. 21 (2008), 1085-1104.

[18] J. H. Bruinier and J. Funke, 'On two geometric theta lifts', Duke Math. J. 125 (2004), 45-90.

[19] J. Bryson, K. Ono, S. Pitman and R. C. Rhoades, 'Unimodal sequences and quantum and mock modular forms', Proc. Natl. Acad. Sci. USA 109(40) (2012), 16063-16067.

[20] Y.-S. Choi, 'The basic bilateral hypergeometric series and the mock theta functions', Ramanujan J. 24 (2011), 345-386.

[21] G. Coogan and K. Ono, 'A $q$-series identity and the arithmetic of Hurwitz-zeta functions', Proc. Amer. Math. Soc. 131 (2003), 719-724.

[22] L. Dragonette, 'Some asymptotic formulae for the mock theta series of Ramanujan', Trans. Amer. Math. Soc. 72 (1952), 474-500.

[23] F. Dyson, 'Some guesses in the theory of partitions', Eureka 8 (1944), 10-15.

[24] N. J. Fine, Basic Hypergeometric Series and Applications, Math. Surveys and Monographs, 27 (American Mathematical Society, Providence, 1988).

[25] A. Folsom, 'Mock modular forms and $d$-distinct partitions', preprint.

[26] G. Gasper and M. Rahman, Basic Hypergeometric Series, Ency. Math. and App., 35 (Cambridge University Press, Cambridge, 1990).

[27] B. Gordon and R. McIntosh, 'A survey of classical mock theta functions', in Partitions, $q$-series and Mmodular Forms, Dev. Math., 23 (Springer, New York, 2012), 95-144.

[28] M. Griffin, K. Ono and L. Rolen, 'Ramanujan's mock theta functions', Proc. Natl. Acad. Sci., USA 110 (2013), 5765-5768.

[29] K. Hikami, 'Quantum invariant for torus link and modular forms', Comm. Math. Phys. 246 (2004), 403-426.

[30] D. S. Kubert and S. Lang, Modular Units (Springer, Berlin, 1981).

[31] R. Lawrence and D. Zagier, 'Modular forms and quantum invariants of 3-manifolds', Asian J. Math. 3 (1999), 93-108.

[32] J. Lovejoy and K. Ono, 'Hypergeometric generating functions for the values of Dirichlet and other L-functions', Proc. Natl. Acad. Sci., USA 100 (2003), 6904-6909. 
[33] K. Mahlburg, 'Partition congruences and the Andrews-Garvan-Dyson crank', Proc. Natl. Acad. Sci. USA 105 (2005), 15373-15376.

[34] E. T. Mortenson, 'On the dual nature of partial theta functions and Appell-Lerch sums', preprint. arxiv: 1208.6316.

[35] K. Ono, 'Unearthing the visions of a master: harmonic Maass forms and number theory', Proc. 2008 Harvard-MIT Current Developments in Mathematics Conf., (2009), Somerville, Ma., 347-454.

[36] H. Rademacher, Topics in Analytic Number Theory, Die Grund. der math. Wiss., Band, 169 (Springer, New York-Heidelberg, 1973).

[37] R. C. Rhoades, 'Asymptotics for the number of strongly unimodal sequences', Int. Math. Res. Not., to appear.

[38] R. C. Rhoades, 'A unified approach to partial and mock theta functions', Math. Res. Lett., to appear.

[39] L. Rogers, 'On two theorems of combinatory analysis and some allied identities', Proc. Lond. Math. Soc. (2) 16 (1917), 315-336.

[40] G. N. Watson, 'The final problem: an account of the mock theta functions', J. Lond. Math. Soc. 2(2) (1936), 55-80.

[41] D. Zagier, 'Vassiliev invariants and a strange identity related to the Dedekind eta-function', Topology 40 (2001), 945-960.

[42] D. Zagier, 'Ramanujan's mock theta functions and their applications [d'aprés Zwegers and Bringmann-Ono]', Sém. Bourbaki (2007/2008), Astérisque, No. 326, Exp. No. 986, vii-viii, (2010), 143-164.

[43] D. Zagier, 'Quantum modular forms', in Quanta of Maths: Conference in Honor of Alain Connes, Clay Mathematics Proceedings, 11 (American Mathematical Society, Providence, 2010), 659-675.

[44] S. Zwegers, 'Mock $\vartheta$-functions and real analytic modular forms', in q-series with Applications to Combinatorics, Number Theory, and Physics, Contemporary Mathematics, 291 (eds. B. C. Berndt and K. Ono) (American Mathematical Society, 2001), 269-277.

[45] S. Zwegers, Mock Theta Functions, Ph.D. Thesis (Advisor: D. Zagier), (Universiteit Utrecht, 2002).

[46] S. Zwegers, 'Multivariable Appell functions', preprint. 